\title{
Recurrent Renovascular Hypertension due to Giant Liver Cyst
}

\author{
Andrew W. Michael, MD, Peter F. Rovito, MD, Peter V. Rovito, BS \\ Department of Surgery, Lehigh Valley Health Network, Allentown, PA, USA (Dr. Michael). \\ Department of Surgery, Sacred Heart Hospital, Allentown, PA, USA (Drs. Rovito and Rovito).
}

\begin{abstract}
Introduction: Renovascular hypertension is a rare but treatable cause of refractory hypertension and is usually the result of fibromuscular dysplasia or atherosclerotic disease. Infrequently, renovascular hypertension is caused by external compression due to enlarged adjacent structures, such as lymph nodes or adrenal tumors.

Case Description: We present an unusual case of a giant liver cyst that compressed the renal artery and caused subsequent renovascular hypertension that was treated with multiple drainage procedures, with eventual resolution of the patient's symptoms.

Discussion: The application of laparoscopic cyst drainage with adjuncts such as drain placement and sclerosing agents is useful for complete long-term resolution of large hepatic cysts. Resolution of the patient's hypertension indicates renovascular hypertension secondary to the cyst as the etiology of her symptoms.
\end{abstract}

Key Words: Hepatic, Cyst, Renovascular hypertension, Drainage.

Citation Michael AW, Rovito PF, Rovito PV. Recurrent renovascular hypertension due to giant liver cyst. CRSLS e2014.00240. DOI: 10.4293/JSLS.2014.00240.

Copyright (c) 2015 by SLS, Society of Laparoendoscopic Surgeons. This is an open-access article distributed under the terms of the Creative Commons Attribution-Noncommercial-ShareAlike 3.0 Unported license, which permits unrestricted noncommercial use, distribution, and reproduction in any medium, provided the original author and source are credited.

Address correspondence to: Andrew W. Michael, MD, 4221 Waterford Dr, Center Valley, PA 18034, USA. Telephone: (909) 319-4255, E-mail: Dr.Drew@gmail.com

\section{INTRODUCTION}

Renovascular hypertension (RVH) is an uncommon cause of hypertension in unselected groups of patients with mild to moderate hypertension. The cause is an occlusive lesion of the renal artery. The estimated prevalence of renovascular etiologies of hypertension range from $0.6 \%$ to $3 \%$. In referral clinics for severe hypertension, the rate of RVH may exceed $20 \%$ of patients. ${ }^{1}$ The most common causes of $\mathrm{RVH}$ are atherosclerotic disease and fibromuscular dysplasia. Other common etiologies are acute embolic disease, aortic dissection, and renal artery trauma. External compression on the renal arteries is rarely a cause of symptomatic RVH. Most cases of RVH caused by external compression are caused by tumors in adjacent structures such as lymph nodes and adrenal tumors. ${ }^{2,3}$ Rarely, a distant tumor can grow so large that it will displace the kidney and impinge on the renal artery. Case reports of large soft tissue sarcomas and ovarian masses have been shown to cause RVH in certain cases.,4,5

This article describes a patient in whom a large benign liver cyst developed that caused external compression on her renal artery and subsequent RVH. We also discuss her subsequent treatment.

\section{CASE REPORT}

A 54-year-old woman was seen in the emergency department of our institution in September 2007 complaining of new-onset epigastric fullness and abdominal pain, headaches, and elevated blood pressure. She reported her systolic blood pressure, taken at home, to be "in the 160s." She was found to have new-onset hypertensive crisis with an initial triage systolic/diastolic blood pressure of 202 $\mathrm{mm} \mathrm{Hg} / 112 \mathrm{~mm} \mathrm{Hg}$. Her medical history only consisted of asthma and seasonal allergies for which she was prescribed Singulair (Merck, Whitehouse Station, NJ, USA), 10 mg orally daily, and an albuterol rescue inhaler. Her surgical history consisted of a tubal ligation. Her headache symptoms resolved during this emergency department visit. On physical examination in the emergency department, the patient was found to have no neurologic deficits and some mild epigastric tenderness and fullness. The 
findings of the remainder of a 7-point physical examination were within normal limits. A complete blood count and comprehensive metabolic panel were obtained. The patient was found to have mildly elevated liver function test findings. The aspartate aminotransferase and alanine aminotransferase levels were 106 $\mathrm{U} / \mathrm{L}$ and $114 \mathrm{U} / \mathrm{L}$, respectively. The alkaline phosphatase level was elevated, at $426 \mathrm{U} / \mathrm{L}$, and the total bilirubin level was $1.1 \mathrm{mg} / \mathrm{dL}$. A computed tomography (CT) scan of the abdomen and pelvis with intravenous and oral contrast was performed to evaluate her epigastric fullness and abdominal pain. This showed a large $15.5 \times 10.5-\mathrm{cm}$ liver cyst with mass effect on the right kidney (Figure 1). The patient was discharged from the emergency department while receiving amlodipine/benazepril (Lotrel, Novartis, Basel, Switzerland) with surgical follow-up for the liver cyst.

The patient was seen in the office for follow-up with complaints of dyspnea, persistent abdominal fullness, early satiety, and discomfort and was scheduled to undergo diagnostic laparoscopy and drainage of the liver cyst. Because of her concomitant presentation of this mass with accelerated hypertension, basic metabolic panel and renin and aldosterone levels were obtained. Although the electrolyte levels were within the

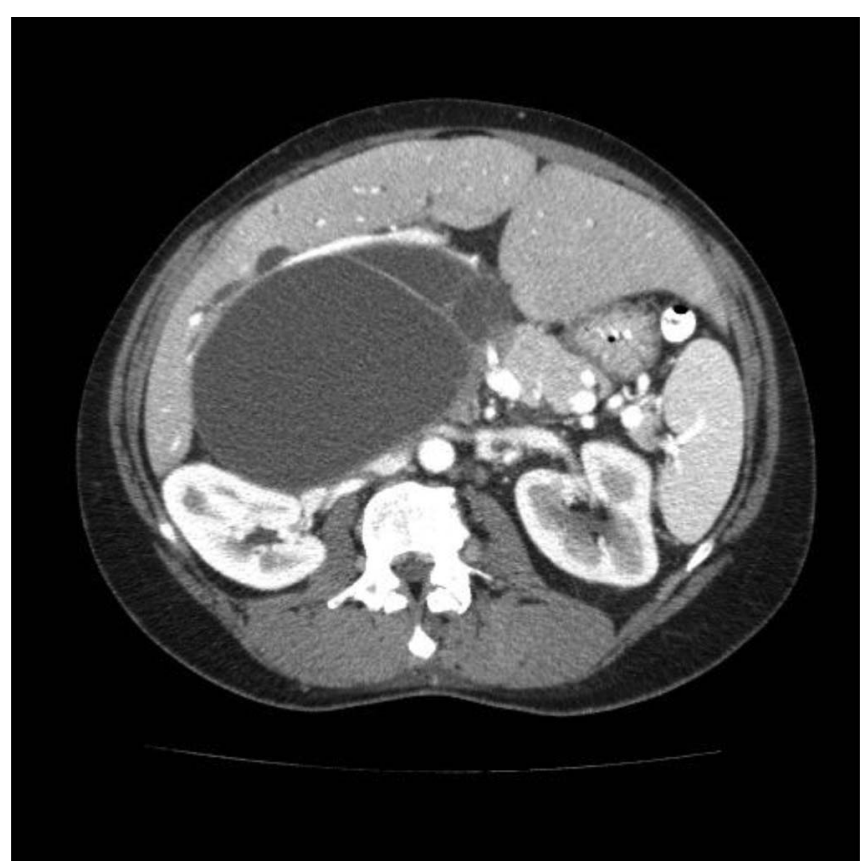

Figure 1. Computed tomography with intravenous and oral contrast exhibiting a large $15.5 \times 10.5-\mathrm{cm}$ liver cyst displacing the right kidney and partially occluding the renal artery. reference values for our institution, the renin level was elevated, at $8.8 \mathrm{ng} / \mathrm{mL}$; the aldosterone level was within the reference range, at $<4 \mathrm{ng} / \mathrm{dL}$ (Figure 2). The patient underwent laparoscopic drainage of the liver cyst about 3 weeks after her initial presentation to the emergency department. The cyst was aspirated, yielding $1300 \mathrm{~mL}$ of serous fluid, and a partial hepatic cystectomy was performed. Both the cyst wall and fluid were submitted to the pathology department for analysis. The cyst wall was curetted to facilitate scarring to prevent cyst re-formation. The patient was discharged on postoperative day 1 ; her systolic/diastolic blood pressure was $112 \mathrm{~mm} \mathrm{Hg} / 64 \mathrm{~mm} \mathrm{Hg}$ while receiving no antihypertensive agents, and her postoperative renin level had normalized to $1.3 \mathrm{ng} / \mathrm{mL}$. The pathologic analysis showed a benign liver cyst wall with no malignant features. The cyst fluid was consistent with serous fluid containing minimal inflammatory cells. The cyst contents were not submitted for an endocrinologic workup.

The patient recovered well from surgery but had a recurrence of her hypertensive dyspnea and abdominal symptoms approximately 1 month postoperatively. A repeat CT scan was performed and showed recurrence of the cyst, measured at $14.2 \times 10.7 \mathrm{~cm}$. The patient was scheduled to undergo repeat laparoscopic drainage. After a similar drainage and partial hepatic cystectomy, an omentopexy was performed with suturing of an omental tongue to the remaining cyst wall. The patient tolerated this procedure well. Again, the cyst wall and fluid specimens were benign, and the renin level dropped from $6.9 \mathrm{ng} / \mathrm{mL}$ to $2.9 \mathrm{ng} / \mathrm{mL}$, with resolution of her hypertension postoperatively.

The patient did well for 3 months; then, over a period of 2 weeks, she again noticed dyspnea, abdominal fullness, and refractory hypertension and was seen again in the office. Her workup findings were similar to the previous findings (ie, CT scan, renin levels), which verified recurrence of the cyst. She was again taken to the operating room. At laparoscopy, a large hepatic cyst was again encountered and drained. At that time, a sclerosing agent of dilute doxycycline $(1 \mathrm{~g}$ in $50 \mathrm{~mL}$ of normal saline) was used to irrigate the cyst cavity and a 19F Blake drain was left in the cyst cavity.

She tolerated the procedure well and was discharged on postoperative day 1 with the drain. A CT scan was performed 3 weeks postoperatively and showed an interval decrease in the cyst size to $6.0 \times 7.2 \times 8.3 \mathrm{~cm}$ (Figure 3). Use of the drain was discontinued 2 months after the last procedure after drainage had ceased altogether. The patient was seen in 


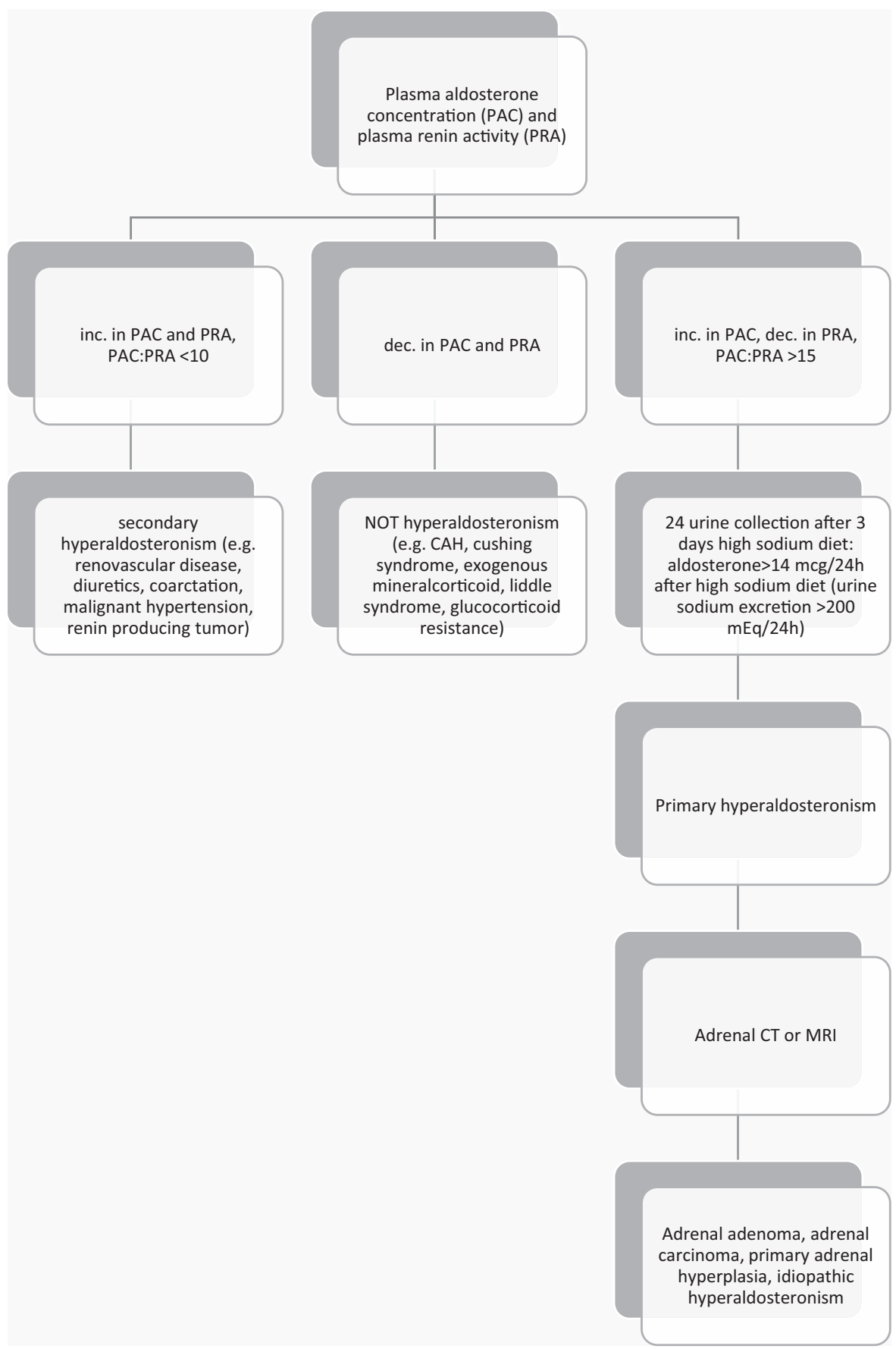

Figure 2. Common renin/aldosterone derangements in hypertensive disorders. $\mathrm{CAH}=$ congenital adrenal hyperplasia; $\mathrm{CT}=\mathrm{computed}$ tomography; dec $=$ decrease; inc $=$ increase; $\mathrm{MRI}=$ magnetic resonance imaging.

the office and underwent CT scanning 10 months after her third operation, which showed nearly complete resolution of the cyst, measuring $2.7 \times 3.7 \mathrm{~cm}$ (Figure 4). She had had complete resolution of her hypertension as well as her abdominal and respiratory symptoms.

\section{DISCUSSION}

In the 19th century, Richard Bright suggested a causal relationship between renal disease and hypertension. Also in the 19th century, Tigerstedt and Bergman performed 


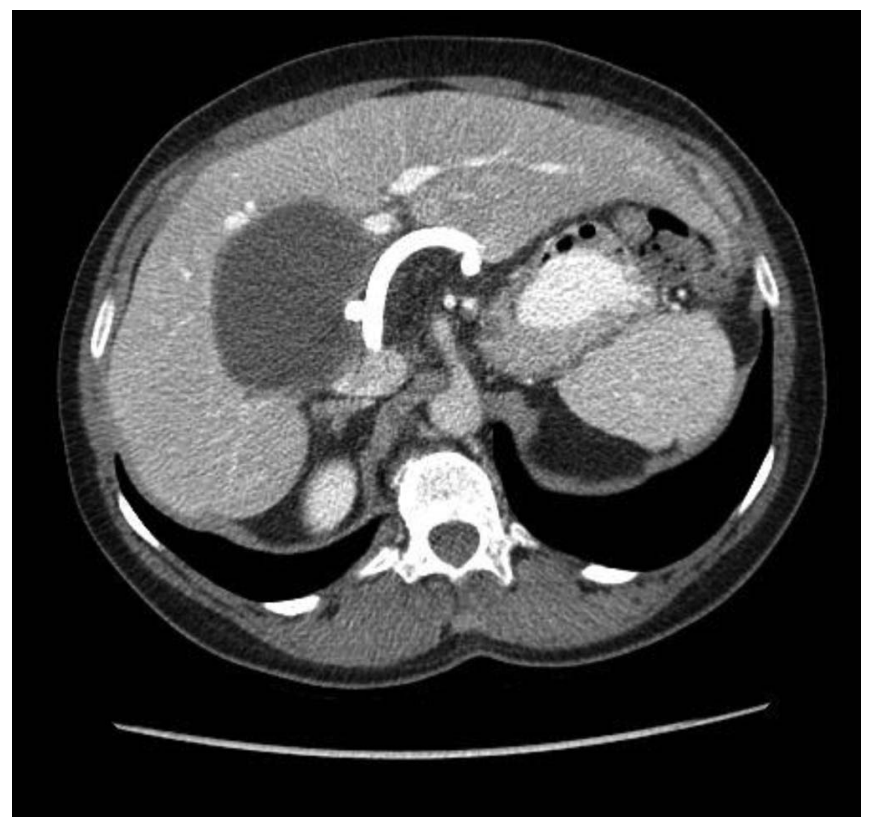

Figure 3. Computed tomography with intravenous contrast exhibiting interval decrease in cyst size to $6 \times 7.2 \times 8.3 \mathrm{~cm}$ with surgical drain in place.

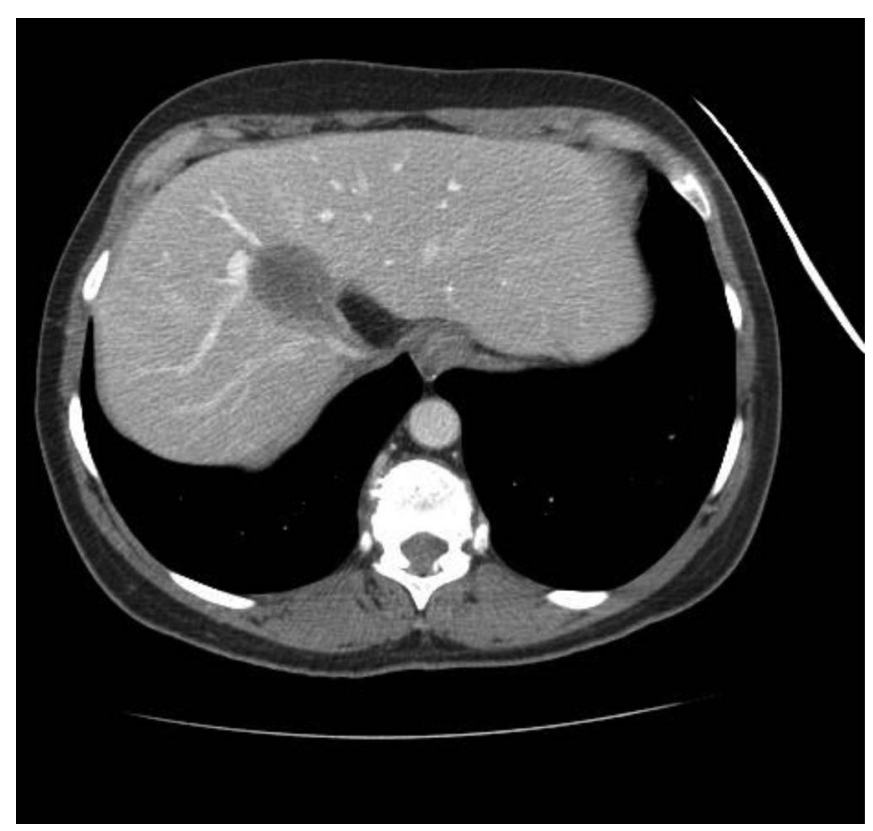

Figure 4. Computed tomography with intravenous contrast exhibiting nearly complete resolution of previously imaged cyst to $2.7 \times 3.7 \mathrm{~cm}$ with interval removal of surgical drain.

experiments using rabbits and injecting them with a substance found in the renal cortex that led to an increase in blood pressure. This substance was named renin. Approximately 100 years later, Harry Goldblatt clearly

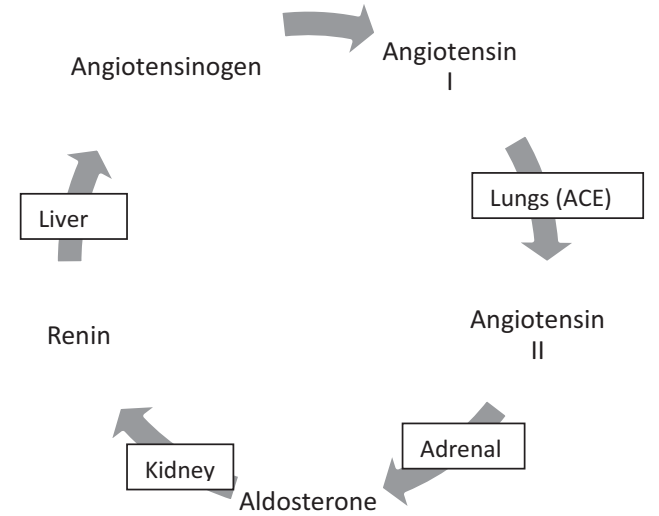

Figure 5. Renin-angiotensin-aldosterone system. ACE = angiotensin converting enzyme.

demonstrated the renal etiology for hypertension. ${ }^{6}$ In his seminal studies, Goldblatt experimented with dogs by reducing the perfusion to the kidney. He observed that this can cause a sustained increase in arterial pressure. This discovery was later confirmed by 2 groups, led by Braun-Menendez and Page. ${ }^{7,8}$ In an attempt to purify renin, they discovered angiotonin, which was later renamed angiotensin. Later on, Skeggs et al ${ }^{9}$ discovered that this peptide existed in 2 forms as angiotensin I and angiotensin II. Angiotensin II stimulates the release of an adrenal cortical hormone called aldosterone. This process in its entirety was called the renin-angiotensin-aldosterone system (Figure 5).

The most common causes of RVH are atherosclerotic disease and fibromuscular dysplasia. Other common etiologies are acute embolic disease, aortic dissection, and renal artery trauma. Less commonly, external compression from adjacent tumors or large distant tumors can cause symptomatic RVH. A review of the literature shows that no such mass arising from the liver has been reported to cause extrinsic compression of the renal artery causing $\mathrm{RVH}$. It is not uncommon for a hepatic neoplasm to cause local mass effect symptoms such as epigastric fullness, discomfort, early satiety, and biliary obstruction. It is, however, uncommon for hepatic neoplasms to cause symptoms related to compression of the visceral and retroperitoneal organs, such as the small bowel, colon, or blood vessels. Our patient had epigastric fullness and discomfort, which were expected with a hepatic cyst of this size; however, she also had hypertensive symptoms due to RVH caused by external compression of the hepatic cyst. To establish a unifying diagnosis for both the hypertension and abdominal symptoms and to ensure that there was not a concurrent second cause of RVH, such as 
atherosclerosis, a renin/aldosterone level was obtained before and after surgery. Normalization of renin levels concurrent with resolution of symptoms and hypertension confirmed the cyst as the etiology of the patient's RVH. In addition, when her symptoms returned, her blood pressure and renin levels again were both found to be elevated. After repeat drainage, both the blood pressure and renin levels again returned to normal values. Twice reproducing this correlation makes other causes of secondary hypertension much less likely in this patient.

Complete and long-term symptom resolution in our patient did not occur until a sclerosing agent (doxycycline) was used in conjunction with a drain. Doxycycline has been used as a sclerosing agent for lymphoceles among its other applications. ${ }^{10}$ Once this was applied and a long-term drain (58 days) was used, the patient no longer had a return of her symptoms or hypertension. CT scan in this case confirmed resolution in this final procedure twice. The application of many cyst unroofing and drainage procedures in this case also illustrates the different adjuncts that can be used in difficult-to-resolve cystic lesions.

\section{CONCLUSION}

This report describes a case of a large hepatic cystic lesion presenting with RVH. Drainage of the cyst in this case relieved the patient's symptoms, and recurrence of the cyst correlated well with recurrence of hypertension and return of symptoms. This report also illustrates the use of renin levels to aid in confirming one's clinical suspicion of RVH. In addition, this series of procedures in a single patient illustrates one surgeon's algorithm for proceeding with treatment of a recurrent symptomatic benign hepatic cyst ultimately resulting in its resolution.

\section{References:}

1. Taler S. Resistant hypertension: comparing hemodynamic management to specialist care. Hypertension. 2002;39:982-988.

2. Fujimoto M, Ohya Y, Abe I, Kobayashi K, Hasuo K, Fujishima M. Renovascular hypertension caused by lymph node metastasis in a patient with uterine cervical cancer: case report. Angiology. 1993;44:402-405.

3. Sato T, Sakamoto S, Maebashi M, et al. Hypertension due to combination of pheochromocytoma and unilateral renal ischemia by tumor compression. Jpn Heart J. 1967;8(2):202-208.

4. Khin N, Chitturi S, Karanth N. Severe hypertension due to external compression of unilateral renal artery by retroperitoneal Ewing's sarcoma improved by chemotherapy. Podium presentation at: The Endocrine Society's 95th Annual Meeting \& Expo; June 17, 2013; San Fransisco, CA.

5. Jan F, Roussel L, Lassaily R, Rahmouni A, Levy E, Cachin J. Arterial hypertension due to compression of the renal artery, revealing ovarian cancer [in French]. Presse Med. 1999;28(16): 841-844

6. Peart S. Development of ideas on renovascular hypertension. Semin Nephrol. 2000;20(5):388-393.

7. Goldblatt $\mathrm{H}$. The effect of main artery occlusion of one kidney on blood pressure of dogs. Proc Natl Acad Sci US A. 1976;73(5):1722-1724.

8. Braun-Menendez E, Page I. Suggested revision of nomenclature-angiotensin. Science. 1958;127(3292):242-242.

9. Skeggs L, Dorer F, Kahn J, Lentz K, Levine M. The biochemistry of the renin-angiotensin system and its role in hypertension. Am J Med. 1976;60(6):737-748.

10. Caliendo M, Lee D, Queiroz R, Waldman D. Sclerotherapy with use of doxycycline after percutaneous drainage of postoperative lymphoceles. J Vasc Interv Radiol. 2001;12(1):73-77. 